\title{
COMUNICACIONES BREVES
}

\section{COLERA. CORRELACION DEL DIAGNOSTICO CLINICO CON EL DIAGNOSTICO BACTERIOLOGICO}

\author{
Elizabeth Castañeda*, Rosalba Molineros**, Roberto Castillo***.
}

\begin{abstract}
Después de haber establecido, por métodos bacteriológicos, la amplia circulación del Vibrio cholera 01 en las poblaciones de Tumaco y Salahonda, se determinó la sensibilidad y especificidad de la definición de caso clínico de cólera. Con este fin estudiamos 185 pacientes que acudieron a los centros hospitalarios a consultar por diarrea. De acuerdo con el cuadro clínico, se anotó la impresión diagnóstica de cólera o de otro tipo de enfermedad diarreíca aguda y se remitieron las muestras de materia fecal al laboratorio, donde se procesaron de acuerdo con la metodología estandarizada. El diagnóstico clínico fue de cólera en 152 de los 185 pacientes y en el laboratorio se aisló Vibrio cholera 01 en 125 de las 185 muestras. El análisis de los datos demostró para el diagnóstico clínico una sensibilidad del $91,2 \%$, una especificidad del $36,7 \%$ y valores predictivos positivo y negativo del $75 \%$ y $67 \%$ respectivamente. Confirmamos la utilidad de la definición de caso en el área en estudio.
\end{abstract}

En el documento Guias para el Control de Cólera (1) la Organización Mundial de la Salud (OMS), establece el papel del laboratorio de Bacteriología Clínica durante una situación epidémica: si bien, el tratamiento exitoso del cólera no depende de los resultados de los exámenes de laboratorio, el análisis de las muestras provenientes de los primeros casos sospechosos es esencial para confirmar la presencia del agente etiológico y permitir de esa manera movilizar los recursos y determinar las características epidemiológicas del brote (1).

En el momento en que es confirmada en una localidad la presencia del Vibrio cholera O 1 no es necesario examinar muestras de todos los casos y los contactos, basándose el diagnóstico, a partir de ese momento, y en esa área en la observación clínica para emplear la definición de caso clínico probable que debe establecerse en una situación epidémica $(1,2)$.
Durante la actual epidemia de cólera y después de haber establecido la circulación del V. cholera 01 , toxigénico, subtipo Inaba, biotipo El Tor, en Tumaco y Salahonda (Nariño), primeras poblaciones afectadas por la epidemia en nuestro país, decidimos determinar la sensibilidad y especificidad de la definición de caso clínico probable que había sido establecida por el Comité Nacional de Vigilancia y Control del Cólera. Estudiamos todos los pacientes que acudieron a consultar por un cuadro diarreíco, al Hospital San Andrés de Tumaco y al Centro de Salud de Salahonda en el tiempo comprendido entre el 8 y el 26 de abril de 1991. De acuerdo con el cuadro clínico y con la definición de caso probable de cólera, en el cual se consideraba todo paciente con deposiciones diarreícas líquidas de inicio súbito, grave o leve, los médicos anotaron en la hoja de remisión de muestras al laboratorio su impresión diagnóstica de cólera o de otro tipo de enfermedad

\footnotetext{
* Grupo de Microbiología del Instituto Nacional de Salud.

** Hospital San Andrés de Tumaco

${ }^{\star * \star}$ Hospital de Salahonda, Francisco Pizarro. Servicio Seccional de Salud de Nariño
} 
diarreíca aguda; también enviaron al laboratorio muestras de la materia fecal en recipientes limpios o impregnaron en ella escobillones tratados con carbón los cuales remitieron en el medio de transporte Cary-Blair. Las muestras fueron procesadas en el laboratorio para el aislamiento e identificación de V. cholerae $\mathbf{0 1}$, siguiendo los procedimientos previamente estandarizados en el laboratorio de Referencia Nacional (3). La sensibilidad y especificidad fue determinada empleando una tabla de $2 \mathrm{X} 2$ y la significancia de los resultados fue analizada con la prueba de contraste de Mac Nemar (4).

El diagnóstico clínico de cólera fué establecido en 152 de los 185 pacientes estudiados y en el laboratorio se aisló V.cholerae 01 en 125 de las 185 muestras correspondientes al número de pacientes. En la Tabla 1 se expresa el análisis de los datos el cual se interpreta que con una prevalencia de la entidad del $67,5 \%$ en las poblaciones estudiadas, la sensibilidad del diagnóstico clínico frente al diagnóstico bacteriológico fué del 91,2\%, la especificidad del $36,7 \%$, y los valores predictivos postivo y negativo del 75 y $67 \%$ respectivamente $(p<0.01)$.

TABLA 1.

CORRELACION DEL DIAGNOSTICO CLINICO CON EL DIAGNOSTICO BACTERIOLOGICO

\begin{tabular}{|c|c|c|c|c|c|}
\hline \multirow{4}{*}{$\begin{array}{l}\text { Diagnóstico } \\
\text { clínico }\end{array}$} & \multicolumn{4}{|c|}{ Diagnóstico bacteriológico } & \\
\hline & & + & - & total & \\
\hline & + & 114 & 38 & 152 & \\
\hline & - & 11 & 22 & 33 & \\
\hline & tota & 125 & 60 & 185 & \\
\hline & $\begin{array}{l}S= \\
E=\end{array}$ & $\begin{array}{l}1,2 \% \\
6,7 \% \\
\text { alenci }\end{array}$ & $=6$ & $\begin{array}{l}\text { PV }= \\
\text { NV }= \\
5 \%\end{array}$ & $\begin{array}{l}=75 \% \\
=67 \% \\
p<0.01\end{array}$ \\
\hline
\end{tabular}

Al estratificar los datos por grupos de edad, en los 175 pacientes de los cuales teníamos información, en menores de 10 años $(n=39)$ y mayores $(n=136)$ encontramos que la sensibilidad y especificidad del diagnóstico clínico fueron mayores en el primer grupo ( $95 \%$ y $68,4 \%$ ) comparados con el segundo ( $90,2 \%$ y $17,6 \%)$. No se anotaron diferencias significativas al estratificar por sexo, obteniéndose sensibilidades del $94,5 \%$ y $86,5 \%$ y especificidades del $32,3 \%$ y $41,4 \%$.

En nuestro estudio, sólo el $75 \%$ de los casos que cumplían los criterios clínicos de caso de cólera fueron confirmados por aislamiento del agente. Esto nos indica que a pesar de la alta sensibilidad de la definición, la especificidad fue muy baja, lo cual hizo necesario un ajuste a la definición de caso; esta definición fue revisada a medida que la epidemia avanzó en nuestro país. El incremento en la especificidad de la definición clínica en el grupo de pacientes con diarrea menores de 10 años se explica al considerar que la enfermedad diarreíca aguda es motivo de consulta mayor en este grupo.

Vigilar la definición de caso es una de las funciones del laboratorio durante una epidemia, el lograr un valor predictivo alto para esta definición permite que los pacientes que llenen los criterios establecidos se consideren como pacientes de cólera, aún sin tener confirmación por el laboratorio. Esto a su vez, permite conocer la situación real del alcance de la epidemia y le permite al laboratorio realizar las otras labores que le corresponden, tales como determinar la eficacia de las medidas de control, participar en el programa de control de calidad que debe ser establecido por el laboratorio nacional de referencia y vigilar la susceptibilidad antimicrobiana de las cepas aisladas.

\section{SUMMARY}

During the actual outbreak of cholera in Colombia and after being established the bacteriologic confirmation of Vibrio cholera 01 in the areas of Tumaco and Salahonda (Nariño), we tried to determine the correlation between the case definition of cholera and bacteriological diangosis. We studied 185 patients: clinical diagnosis was established in 153 and V. cholerae 01 was recovered in 125 . The sensibility for clinical diagnosis was $91.2 \%$ the specifity $36.7 \%$ and the positive and negative predictive values were 75 and $67 \%$ respectively. Our data allowed us to confirm the utility of the case definition in the area under study. 


\section{AGRADECIMIENTOS}

Al Doctor Fernando de la Hoz, por su sugerencias en el análisis de los datos. Al estadístico Alberto Serrano por el análisis de significancia y a la bacterióloga Marcela Escalante por su colaboración en el procesamiento de las muestras.

\section{REFERENCIAS}

1. WHO. Programme for Control of Diarrhoeal Disease.
Guidelines for cholera control. 1986: WHO/CDD/SER/ 80.4 REV.1

2. Vugia DJ,Koehler JE,Ries AA.Surveillance for epidemic cholera in the Americas: an assessment. MMWR 1992; 41(SS-1): 27.

3. Restrepo M,Castañeda E, Rivas F, Podlesky E, Urrego G.Cólera. Serie de Notas e Informes Técnicos No 19. Instituto Nacional de Salud. 1991.

4. Lehman. Non Parametric Statistical Methods Based Remkg. Mc Graw Hill. New York. 1976. 